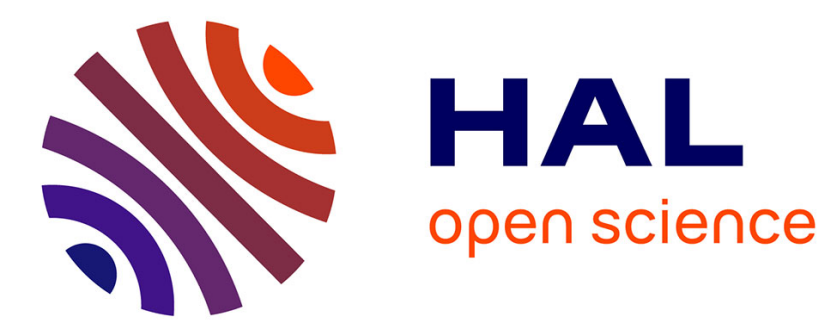

\title{
Forwarding by Retransmission in IEEE 802.11
}

\author{
Lucien Loiseau, Nicolas Montavont, Xavier Lagrange
}

\section{To cite this version:}

Lucien Loiseau, Nicolas Montavont, Xavier Lagrange. Forwarding by Retransmission in IEEE 802.11. ANTS 2013: 7th IEEE International Conference on Advanced Networks and Telecommunication Systems, Dec 2013, Chennai, India. hal-00942322

\section{HAL Id: hal-00942322 \\ https://hal.science/hal-00942322}

Submitted on 5 Feb 2014

HAL is a multi-disciplinary open access archive for the deposit and dissemination of scientific research documents, whether they are published or not. The documents may come from teaching and research institutions in France or abroad, or from public or private research centers.
L'archive ouverte pluridisciplinaire HAL, est destinée au dépôt et à la diffusion de documents scientifiques de niveau recherche, publiés ou non, émanant des établissements d'enseignement et de recherche français ou étrangers, des laboratoires publics ou privés. 


\title{
Forwarding by Retransmission in IEEE 802.11
}

\author{
Lucien Loiseau*, Nicolas Montavont* and Xavier Lagrange* \\ *IMT/TELECOM Bretagne, Université Européenne de Bretagne, Rennes, France \\ \{firstname.lastname\}@telecom-bretagne.eu
}

\begin{abstract}
This paper presents a cooperation-based retransmission mechanism for IEEE 802.11 networks called Forwarding By Retransmission (FBR). This mechanism aims at reducing the mean number of retransmissions in an infrastructure network, or it can be used to forward frames in a small ad hoc network. FBR enables any node that received a frame to participate in the retransmission process, as long as it has a higher probability to reach the destination than the sender. We detail our implementation in NS-2 and analyze the FBR operations in a simple scenario. We show that using only one relay allows reducing the number of retransmissions by $60 \%$.
\end{abstract}

Keywords-IEEE 802.11, cooperation, wireless communication, relays

\section{INTRODUCTION}

The wireless transmission medium is subject to loss due to physical property of the environment separating a source from its destination, path loss, shadowing, or fading effects. The radio medium is unpredictable, and varies over time. Given a source and a destination, some frames may be correctly received while other not. The wireless medium is shared among all stations, and collisions and interferences may also impact performances. In this challenging environment, different standards define how wireless stations can communicate together, such as IEEE 802.11, Bluetooth or LTE to cite only a few. Several modes of communication are proposed, which can be grouped into two main sets. In the infrastructure mode, all exchanges are made with a central entity called an access point (AP). This AP either plays the role of a bridge between the wireless network and the wired network, or it can relay frames from a wireless station to another one. In the ad hoc mode, stations communicate directly with each other. To reach a destination out of its range, a station may use its neighbors as relays, that will forward the frame hop by hop toward the destination. In this case, a routing protocol is usually needed to set up the routes.

A routing protocol for ad hoc network is usually operating in two steps. First, nodes discover their neighbors and establish routes to their destination. We can distinguish two approaches; in proactive protocols, the discovery is made independently from any data traffic. Another approach called reactive protocol consists in determining a specific route only if a source needs to send traffic to a destination. Once the routes are known, the second step is the forwarding process. Data packets are forwarded in unicast, hop by hop up to the destination. These methods do not consider the nature of the wireless transmission medium as described above. It makes the assumption that once a route is discovered, it remains valid and fixed for data transfer. If one hop is not operational anymore (because of a node mobility or another neighbor appears to be more suitable for the fowarding), the routing protocol may adapt the path. However, this adaptation requires a long time to be set up. These approaches rely on the principle of choosing a given hop based on previous performance information. In our proposal, potential relays are selected frame by frame, among all stations that received the frame.

We propose a novel approach for frame relaying in both infrastructure and ad hoc network. In our proposal, a source sends its frames directly to the destination, whether the destination is in its radio range or not. Neighbors that overheard these frames and do not receive the acknowledgement (ACK) from the destination may participate to the retransmission process. For each individual transmission, any receiver can participate in the retransmission process if it allows making progress toward the destination. We call this opportunistic relaying because a source can not know in advance which nodes will receive its transmission and potentially act as a relay. We apply our mechanism in the IEEE 802.11 standard, with as few as possible modification to the standard. We need to change a node behavior with regards to the overheard frames: a receiver will process every frame, even those for which if it is not the destination.We also slightly modify the acknowledgement system, by adding two types of acknowledgement: one is the passive acknowledgement where the retransmission of a frame by a neighbor is considered as an acknowledgement. The other one is the delayed acknowledgement, when a node receives an unexpected but legitimate ACK for a queued frame.

The remainder of this paper is organized as follows. Section II introduces our approach and explains how it can apply to both infrastructure and ad hoc networks. Section III formally presents the protocol and section IV describes its implementation in NS2 and preliminary results. Section $\mathrm{V}$ concludes the paper. 


\section{RELATED WORK AND APPLICABILITY}

The wireless transmission medium is broadcast by nature, so any node in the radio range of a source may receive its frames, even if they are not the intended destination. While traditional routing protocols predefine a next hop for routing a frame through multiple hops, we propose to take advantage of opportunistic frame reception by any node. We assume that the set of receiving nodes is different from one transmission to another, so instead of selecting a (fixed) next hop, we aim at selecting a forwarder among those which received a frame. The selection process is decentralized and computed individually on each receiver. Each potential forwarder will compete to retransmit a frame. Once a station wins the contention and retransmits a frame, all potential forwarders (including the source itself) must consider this retransmission as an acknowledgement. The selected forwarder is then responsible for the correct reception of the frame to the next hop or the destination.

This mechanism relies on a discriminant that allows a receiver to know whether it is potentially a better retransmitter than the sender. This discriminant can be any link metric that can estimate the link quality between a node and a given destination. For example, it can be the RSSI or the ETX. In the following, we develop how our mechanism can operate in infrastructure or ad hoc networks.

\section{A. Relaying in infrastructure topologies}

In the infrastructure mode, a node sends all its frames to an AP. So a cell is defined as the set of nodes that are able to communicate (send and receive) frames with a given AP. In order to optimize the cell size, different modulations can be selected to adapt to the link quality between the AP and a given node. For example, a node with a low signal strength may choose a robust modulation scheme that allows repairing the channel errors. On the contrary, a node that is close to the AP may use a less robust modulation scheme that allows higher data rate. In $802.11 \mathrm{~g}$, data rates can vary from $6 \mathrm{Mbps}$ to $54 \mathrm{Mbps}$. Nodes usually select the highest possible data rate depending on their link quality.

Low data rate stations can access the channel as often as high data rate stations, but once they access the medium, they require more time to transmit their frames [1]. For example, transmitting a 1500 byte frame at $6 \mathrm{Mbps}$ requires $2 \mathrm{~ms}$ while it only takes $0.22 \mathrm{~ms}$ at $54 \mathrm{Mbps}$. So even if stations with bad radio conditions are able to connect to an AP, they degrade the performance of all stations in the cell. In recent studies that we conducted in city centers [2], we showed that the signal strength between a mobile station and its AP in community network is quite low, equal or less than $-75 \mathrm{dBm}$ in $80 \%$ of the cases. To avoid low data rate stations to decrease the entire cell performance, stations could cooperate together: high data rate stations could relay frames from stations with low signal strength.

In this context, an AP is periodically sending beacons at $6 \mathrm{Mbps}$. These frames can be used by stations to evaluate their link quality with the AP. By adding this information in their frames, a receiving station is able to determine whether it is in a a better or worst condition to retransmit a frame. Assuming that there is a large density of stations, a station will send its frame at the maximum data rate, whatever its link quality. If the frame is not received by the AP, a receiving station can retransmit the frame to the AP. As shown by [3], a station that retransmits other station frames may increase its own performance (compared to a situation where the source would send its frames at a low data rate without any help from other stations).

\section{B. Relaying in ad hoc networks}

In ad hoc networks, a routing protocol is needed to establish the paths between a source and a destination. Usually the network topology is first discovered and then the paths are computed. For example, the RPL protocol [4] builds a tree where each station determines which other station is its parent toward the root. The network topology is discovered via the DIO messages periodically sent by all stations. This message contains the rank information, i.e., the "distance" between the sending node and the root of the tree. From the DIO sent by all its neighbors, a station can select its parent. The frequency at which the DIO is sent decreases when the topology is stable. In AODV [5], a route request flooded in the network allows discovering a path toward a destination and feed intermediate node routing tables. For each destination, the routing table indicates which neighbor is the next hop toward the destination.

Selecting a parent or the next hop by default is sub-optimal if we take into account that each individual transmission is unique, and each transmission may reach a different set of receivers. By selecting a default parent, a station has to select the neighbor with which it has a reliable link, i.e., the neighbor that will require the minimum number of retransmissions. However, some transmissions may be received by other neighbors than the default parent, including nodes that are closer to the destination. With our mechanism, we enhance such routing protocols by selecting the neighbor that has the better rank with regards to the destination, even if this neighbor may require multi-hops for certain transmissions. Thus, we could split the topology into steps, and use opportunistic relaying inside those steps.

For relative small ad hoc networks, where all stations are able to listen each other beacons, our mechanism applies as well. The link quality with a given destination can be determined by the beacons, and data frames can be sent directly to the destination. If a frame is not acknowledged by the destination, 
because it has not been received, any intermediate node can participate in the retransmission process.

\section{FORWARDING BY RETRANSMISSION}

We propose a lightweight mechanism called Forwarding By Retransmission (FBR) that enhances wireless communication either in an infrastructure network, or an ad hoc network. We provide a retransmission mechanism where a receiving station can participate in retransmitting a frame on behalf of a source if it has a higher probability of successfully transmitting the frame to the destination.

We assume that a destination is sending a beacon frame, which limits the coverage where FBR can be used. Each station can thus compute a link metric with the destination, and include this information in all frames it will send. We used the fourth address field of 802.11 frame to add this information. A source station follows the IEEE 802.11 standard to send a frame. Once it accesses the channel, it sends the frame and waits for the ACK from the destination. If the destination receives the frame, it sends an ACK that is received by all stations, and the transmission is over. Note that the IEEE 802.11 standard recommends to send management frames at the lowest data rate possible. This ensures that the ACK frames are unlikely to be lost. If the destination does not send the ACK, all receivers of the frame compare their link quality with the one indicated in the frame. If their link quality is better than the one in the frame, they will be considered as Potential Forwarder, i.e., they enter in competition to retransmit the frame. At this point of time, the source and all stations that received the frame and have a better link quality than the source are competing to access the channel. The station that had the lowest backoff time, will access the medium and send the frame. This station has now the responsibility of further retransmission if needed. If other potential forwarders and the source receive this retransmission, they will cancel their own retransmission of the same frame. And the same process reproduces with this new transmission, i.e., either the destination receives it and sends back an $\mathrm{ACK}$, or a new retransmission process takes place.

Figure 1 shows all possible scenarios when we consider a source sending a frame to a destination, with an intermediate node (called a relay) in between. We classified the scenarios in three states: state A represents the case where only the source is competing for the medium. This is the case for the initial transmission, or when the relay did not receive the transmission. State B represents the cases when both the source and the relay compete to access the medium. State $\mathrm{C}$ represents the cases when only the relay competes to access the medium. The white boxes (A3, A8, A9, B1.3, B1.9, B2.2, B2.7, B2.8, C2, $\mathrm{C} 7, \mathrm{C} 8$ ) represent a final state, where the transmission is done (the destination received and acknowledged the frame, and the source and the relay do not try to

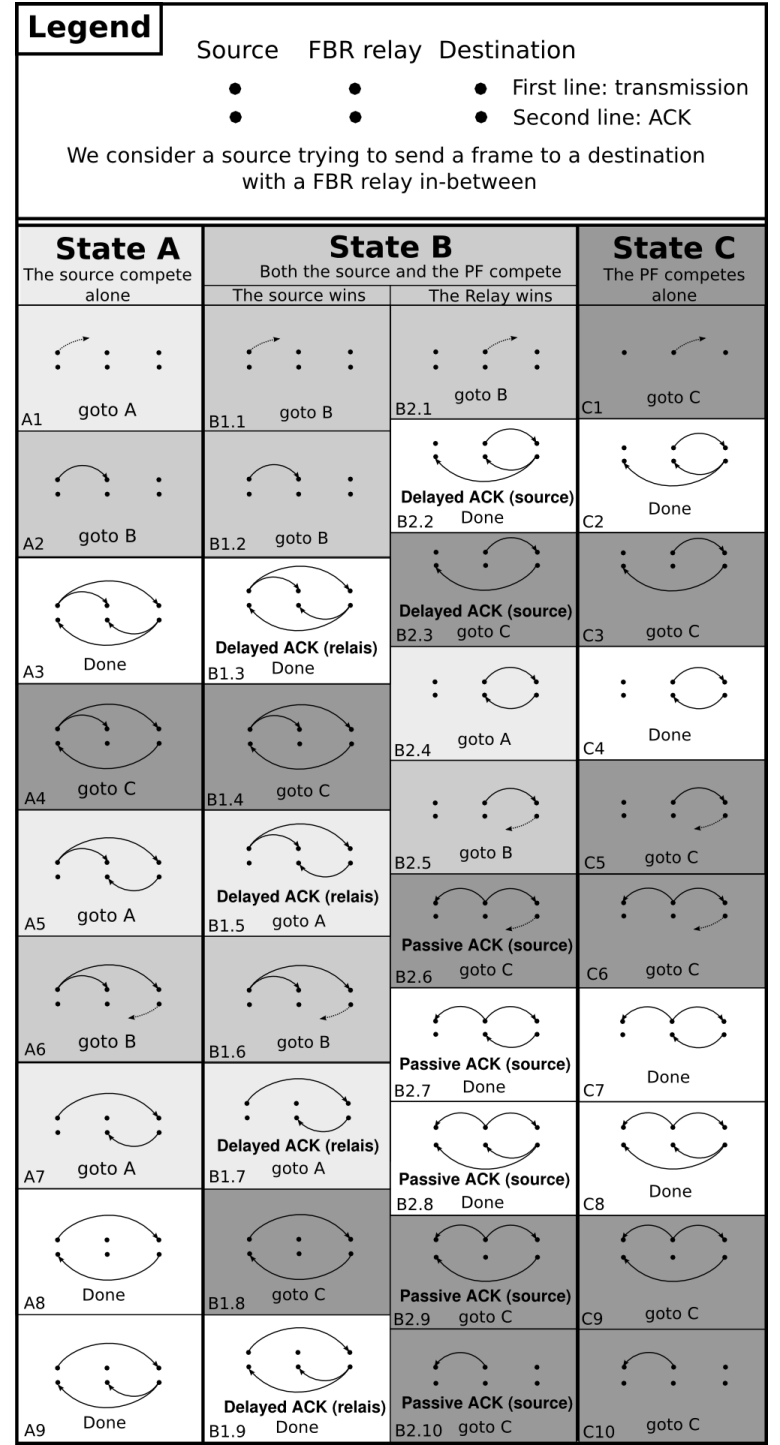

Fig. 1. Scenarios for data transmission

send the frame anymore). Transitions from any state are either to the same state, or forward (e.g., from A to B), except for states B1.5, B1.7 and B2.4 which return to state $\mathrm{A}$. In both $\mathrm{B} 1.5$ and $\mathrm{B} 1.7$ the source won the contention, and retransmitted the frame. In both cases, the destination received the frame, and the relay received the ACK from the destination while the source did not received it. Thus the relay discards the frame from its retransmission queue but the source will try again sending its frame. This is a non usual situation since ACKs are usually received by the sender of a frame, and in any case not caused by our algorithm. The consequence is that the destination will receive a duplicated frame. In B2.4, the relay won the access to the channel and the destination sent an ACK that is only received by the relay, not the source. Note that the source did not receive the retransmission as well. In this case, the source will still try to retransmit its frame. As we will show in the next section, this 


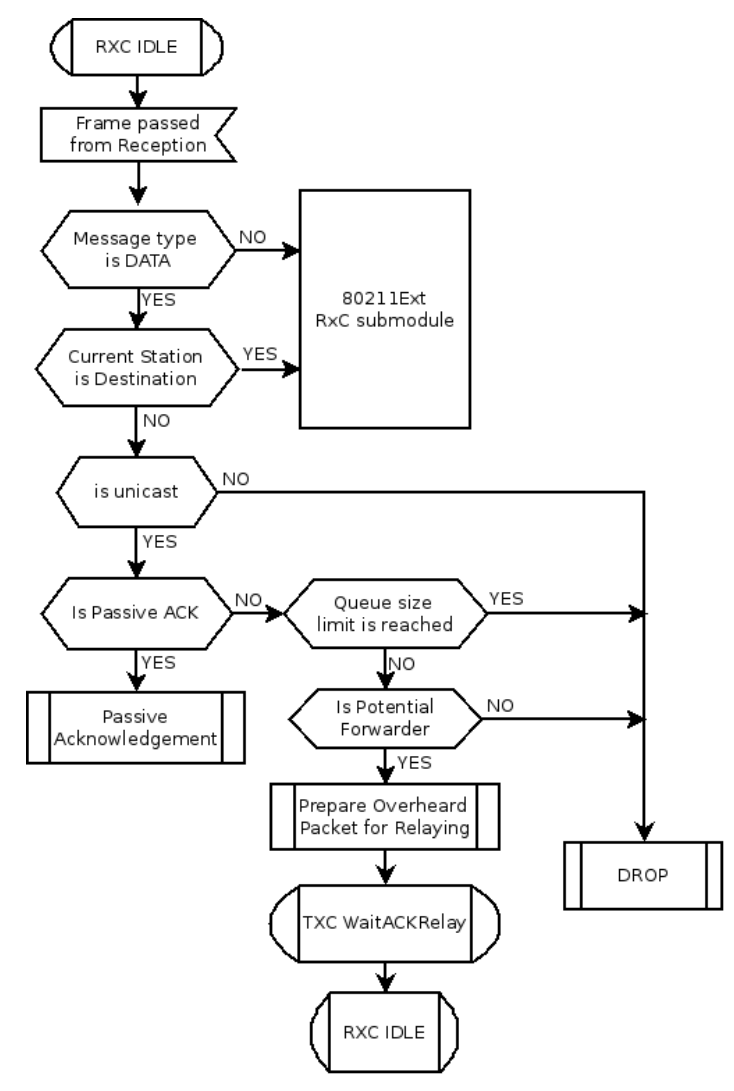

Fig. 2. Modifications to the $\mathrm{RxC}$ submodule

case is also rare and in any case, will only result in a useless retransmission.

In states B1.3, B1.7, B2.2 and B2.3 we observe delayed ACK, which is the reception of an ACK following a successful retransmission by another station. The received ACK still acknowledges the corresponding frame in queue and the receiving stations should discard the queued frame. In states B2.6, B2.9 and B2.10, we consider passive acknowledgement, which is the overhearing of a retransmission by another station of a frame for which a station is waiting to send. For example, in state B2.6, the relay retransmits the frame, and the initial source considers that transmission as an acknowledgement of its previous transmission even though it did not receive the ACK. In this case, the transmission is delegated to the relay. In the next section, we detail the FBR implementation in NS-2 and show preliminary results.

\section{IMPLEMENTATION IN NS-2}

\section{A. NS-2 Implementation}

NS-2 is an open source and well known network simulator written in TCL and $\mathrm{C}++$. We based our implementation on the 802.11Ext module which is one implementation of the IEEE $802.11 \mathrm{~g}$ from the university of karlsruhe [6]. The 802.11Ext module comes with two submodules called mac-80211Ext and phy-80211Ext. We modified mac-80211Ext in order to implement our solution and we improved the physical layer by adding a more realistic packet error rate (PER) model based on Bit Error Rate (BER) sampling found in the literature.

1) Modifying the mac-80211Ext module: This module is composed of several submodules, each of them coordinating the internal parts of the MAC mechanisms. While we left unchanged the Channel State Manager submodule, we modified the Reception Coordination $(\mathrm{RxC})$, the Transmission Coordination (TxC) and the Backoff manager submodules. Figure 2 shows the FBR implementation in $\mathrm{RxC}$, the submodule responsible for handling frames received by a station. In the $R X C_{-} I D L E$ state, a station may receive a frame that can be a data, ACK, RTS or CTS. If the received frame is a data and the receiving station is not the destination, we implemented additional functions. First, we check if the received frame is a frame that the receiving station is currently trying to send. If this is the case and the received frame was sent by a better station, it will be considered as a passive acknowledgement; it shows that the frame has already made progress toward the destination. Thus, the station will remove this frame from its queue. Otherwise, if the frame is not a passive acknowledgement, it might need a retransmission. The receiving station checks if it is a potential forwarder i.e. if it has more chances to reach the destination than the sending station. If so, it prepares the frame for relaying (setting its own signal quality into the fourth address field) and waits for the ACK (state TXC WaitACKRelay).

Figure 3 shows the FBR implementation in TxC, the submodule responsible for managing the frame to be transmitted by the station. We only show the FBR part of the module that starts with the state WaitACKRelay. This state means that the station overheard a unicast data frame sent by a station with a lower signal quality than the current station. If the station receives an ACK before the timer expires, no retransmission is needed and the overheard frame is dropped. If the timer however expires, a retransmission is needed and the station starts a backoff procedure to retransmit the frame (state RELAY PENDING). Then two outcomes are possible. Either the station receives a passive acknowledgement (as explained above) in which case the backoff is cancelled and the frame is dropped. Or the backoff timeouts and the station retransmits the frame, waiting again for an ACK (state WaitACKRelay). This loops until it is acknowledged (passively or with an ACK) or until it reaches the maximum number of retransmissions.

In order to implement a fair queuing between the frames originating from a station and the frames being relayed by this station, we modified the backoff management submodule in order to manage two independent backoffs. Each backoff manages its own contention window size and randomly picks a number of slots (as long as the two backoffs are different to avoid collision). 


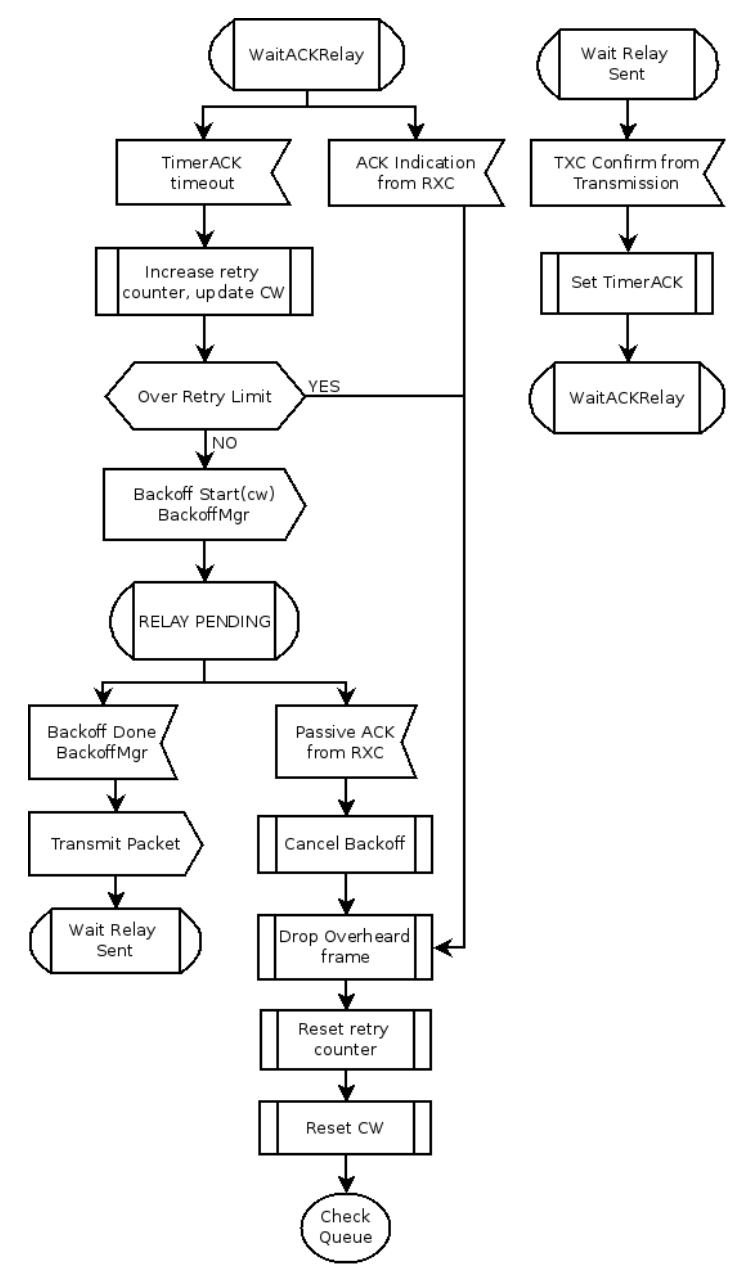

Fig. 3. Modification of the TxC submodule

2) Implementing a realistic Packet Error Rate model: The 80211Ext does not take into account the frame size in the computation of the PER. It provides a threshold algorithm based on the receiving power of a frame to state whether the frame is received or not. Since our algorithm strongly depends on the reception of the ACK, such a limitation would have strongly biased our results as an ACK would have had the same chance of being lost than a 1500 byte frame.

IEEE $802.11 \mathrm{~g}$ uses OFDM to provide various data rates (from 6 to $54 \mathrm{Mbps}$ ). Each data rate has its own characteristics such as the valence (number of bits that one carrier can code), the modulation (BPSK, QPSK, 16QAM or 64-QAM), the coding rate (redundant information to make it more resistant to perturbation) or the hamming distance $d$. The BER is different from one data rate to another as it depends on these characteristics. We used a sampling array found in the literature [7] in order to estimate the BER values. Given the BER, we were able to compute the PER using the following formula:

$$
\left\{\begin{array}{l}
P E R_{1}=\frac{L \cdot B E R}{d}, \\
P E R_{2}=1-(1-B E R)^{L},
\end{array}\right.
$$

$$
P E R=\min \left(P E R_{1}, P E R_{2}\right)
$$

where $d$ is the hamming distance corresponding to the data rate used to transmit the frame and $L$ is the frame size.

\section{B. Validation and Results}

We performed simulations to validate the implementation and to show that FBR improves legacy IEEE 802.11. The scenario consists of one IEEE 802.11 AP, one station acting as a source of traffic and a FBR station in between. The source is located so that its PER with the AP is $33 \%$. This means that over 100 frames sent, 33 will not be received by the access point and will need a retransmission. The FBR node being placed between the source station and the AP, it has a PER lower than $0.01 \%$ with both of them. We performed two runs, one with the FBR mechanism disabled and another one with the FBR algorithm enabled. In both cases, the source sends a CBR (Constant Bit Rate) traffic to the AP at the maximum rate possible, saturating the channel.

Figures 4 and 5 represent the transition probability of the source station when it tries to send a frame. When the source sends a frame for the first time, it goes from the state IDLE to the state WAIT_RX_ACK through a $T X$ transition. This means that the source sent a frame to the access point and is now waiting for an ACK from the AP.

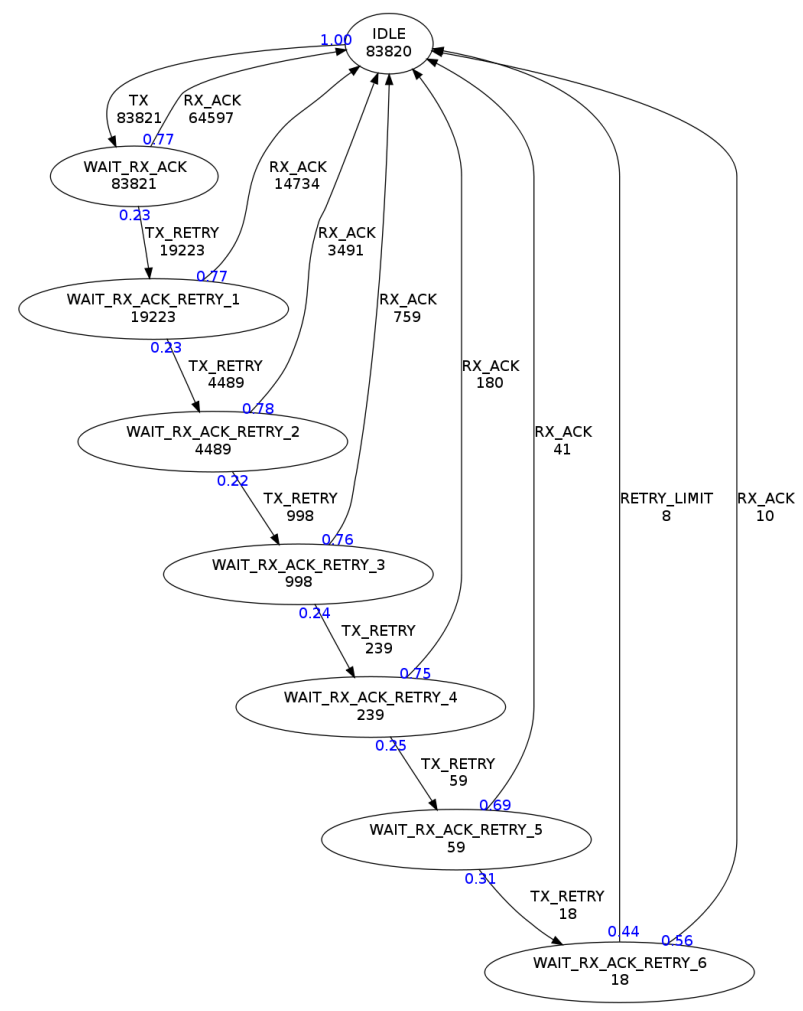

Fig. 4. Transition probability for legacy 802.11 
In the Legacy IEEE 802.11, presented in figure 4, two transitions are possible: one is labelled $R X \_A C K$ and goes back to $I D L E$. This first transition means that the station has received the ACK sent by the AP and thus acknowledges the frame. It goes back to the $I D L E$ state because the retransmission process for this frame is done. The other transition is labelled TX_RETRY and goes to the state WAIT_RX_ACK_RETRY_1. This means that a timeout fired and the station has retransmitted its frame and is now waiting for an ACK. If the transmission failed again, it retransmits the frame and goes to the state WAIT_RX_ACK_RETRY_2, and it goes on until the frame is acknowledged or it reaches the maximum number of retransmissions as shown by the RETRY LIMIT transition.

In the FBR mode shown in figure 5, a third transition is possible from the state WAIT_RX_ACK. This transition is labelled $R X \_D E L A Y E D \_A C K$ and goes back to IDLE. This transition means that the FBR station placed between the source and the AP has overheard the transmission of the source and has decided to retransmit the frame after it has detected that no ACK had been sent. This retransmission made by the FBR node on behalf of the source is immediately followed by an ACK from the AP. Whether it is the overhearing of the retransmission or the reception of its following ACK by the AP, both are considered by the source as a delayed acknowledgement because the frame is being acknowledged (passively or by the ACK) after the standard timeout for which the value is $\operatorname{SIFS}(10 \mu \mathrm{s}$ in IEEE $802.11 \mathrm{~g})$.

We observe that the mean number of transmissions (ETX) decreased from 1.30 without FBR, to 1.26 when FBR is enabled. In both cases, the source succeeds in transmitting a frame in the first attempt in $67 \%$ of the times (as expected since its PER is $33 \%$ ). If we focus on the frames that actually needed a retransmission, we can see that without FBR, the retransmission overhead is $30 \%$ which means that for 100 frames that needed to be retransmitted, 130 transmissions have been necessary. The retransmission overhead is only $12 \%$ with FBR enabled which means that we reduced the number of retransmissions by $60 \%$. If the relay would have won every contention, we woud have needed only one retransmission for each unacknowledged frame, reaching $100 \%$ of reduction, because the relay almost never fails its transmission. Even though the relay does not win every contention, the contention window of the relay is always lower than the source's. The source increases its contention window at each retransmission giving the relay better chance to win the contention. Moreover, as the number of retransmissions is reduced, it creates more opportunities for the source to send new data. Indeed, less retransmission means that the contention window size will be lower on average, reducing the backoff time as well. As a consequence, the source is able to send more frames, reaching $7.03 \mathrm{Mbps}$ with FBR against 6.7Mbps without FBR.

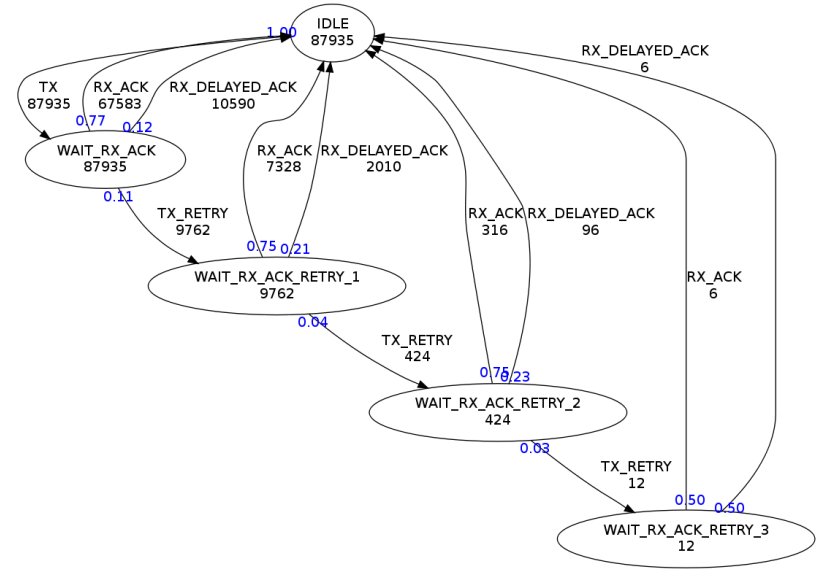

Fig. 5. Transition probability for FBR

\section{CONCLUSION}

We described a new mechanism called FBR designed for IEEE 802.11 networks for frame relaying. FBR allows, under certain conditions, surrounding nodes to retransmit a frame on behalf of the source. We detailed the FBR implementation into the Network Simulator NS-2 which is two fold. Firstly we modified the Mac-80211Ext module so that FBR can be enabled and secondly, we enhance the physical layer in order to take into consideration the size of the frame in the computation of the Packet Error Rate. Finally we provided a simulation analysis using transition diagram that shows how FBR works and how it outperforms the legacy IEEE 802.11 mechanism.

\section{REFERENCES}

[1] M. Heusse, F. Rousseau, G. Berger-Sabbatel, and A. Duda, "Performance anomaly of 802.11 b," in INFOCOM 2003. Twenty-Second Annual Joint Conference of the IEEE Computer and Communications. IEEE Societies, vol. 2, pp. 836843, IEEE, 2003.

[2] G. Castignani, A. Blanc, N. Montavont, and A. M. Lampropulos, "Urban 802.11 Community Networks for Mobile Users: Current Deployments and Prospectives," Mobile Networks \& Applications, pp. 1 - 12, august 2012.

[3] P. Bahl, R. Chandra, P. Lee, V. Misra, J. Padhye, D. Rubenstein, and Y. Yu, "Opportunistic use of client repeaters to improve performance of wlans," IEEE/ACM Transactions on Networking (TON), vol. 17, no. 4, pp. 1160-1171, 2009.

[4] IETF, "Rpl: Ipv6 routing protocol for low-power and lossy networks," 2012.

[5] C. Perkins, E. Royer, and S. Das, "Rfc 3561 ad hoc ondemand distance vector (aodv) routing," tech. rep., 2003.

[6] Q. Chen, F. Schmidt-Eisenlohr, D. Jiang, M. Torrent-Moreno, L. Delgrossi, and H. Hartenstein, "Overhaul of ieee 802.11 modeling and simulation in ns-2," in Proceedings of the 10th ACM Symposium on Modeling, analysis, and simulation of wireless and mobile systems, pp. 159-168, ACM, 2007.

[7] L. Miller, "Validation of 802.11 a/uwb coexistence simulation," national institute of standards and technology (NIST), WCTG white paper, 2003. 